\section{Kompass \\ Autoimmun}

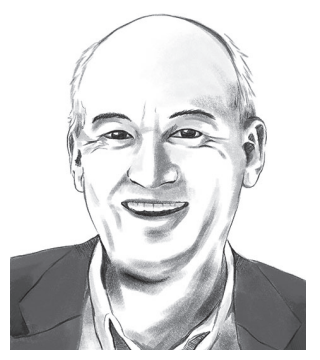

Harald Walach

Poznan/Berlin,

Polen/Deutschland

Kompass Autoimmun 2020;2:92

DOI: 10.1159/000507772

\title{
Die wundersame Zeitvermehrung
}

«Zeit ist Geld» - das ist einer der dümmsten Sprüche der Postmoderne, finde ich. Denn Zeit, nicht Geld, ist unsere wichtigste Ressource und sie ist endlich. Seit Geburt tickt unsere Uhr, irgendwann ist sie unweigerlich abgelaufen. Die Unternehmungen, die wir bis dahin nicht geschafft haben, sind unwiederbringlich versäumt. Und die Gleichung «Zeit = Geld» stimmt sowieso nicht. Denn wer viel Zeit hat, hat meistens wenig Geld, und wer viel Geld hat, hat meistens sehr wenig Zeit. Der größte Unterschied von Zeit und Geld ist aber folgender: Während man Geld fast beliebig vermehren kann - die Banken, die Mafia und viele Neureiche machen uns das ja täglich vor - kann man Zeit eben nicht vermehren, so scheint es.

Ich mache mich jetzt trotzdem an das scheinbar Unmögliche und verrate Ihnen meinen Trick zur Vermehrung der Zeit. Also, hier kommt der Trick: Die wunderbare Zeitvermehrung gelingt, wenn wir es schaffen, alles mit «einem Sinn» zu tun. Mit ungeteilter Aufmerksamkeit, ohne Ablenkung, ohne auch nur im hintersten Winkel unseres Bewusstseins zu denken «ich könnte doch jetzt eigentlich auch noch schnell...». Und wenn wir bemerken, dass es im hintersten oder vordersten Winkel unseres Bewusstseins spuckt, etwa «diese idiotische Sitzung geht mir jetzt einfach zu lang» oder «dieser Mensch mit seinem wiederkehrenden Gejammer geht mir auf die Nerven», dann müssen wir aktiv werden.
Zum Beispiel aufstehen und sagen: «Werte Kolleginnen und Kollegen, ich kann aus religiösen Gründen nicht an Sitzungen teilnehmen, die länger als 60 Minuten dauern.» Oder: «Herr Müller, ich verstehe ja, dass Sie in einer schwierigen Situation sind, aber ich glaube, das haben wir letzte Woche schon mal besprochen. Gibt es was Neues?» Dann müssen wir nämlich nicht, wenn die 2-stündige Sitzung aus ist oder der logorrhoeische Herr Müller seine Geschichte zu Ende lamentiert hat, das Gefühl haben, jetzt aber ganz schnell die verlorene Zeit aufholen zu müssen, indem wir E-Mails, Telefonate und Mitarbeitergespräche gleichzeitig abhalten.

\section{Multitasking ist eine der größten} Illusionen des Computerzeitalters und auch einer der größten Zeitfresser.

Denn das ist der größte Selbstbetrug, beim Versuch Zeit zu gewinnen: Die Illusion, wir könnten Multitasking. Können wir nicht. Nicht einmal Computer können das. Das sieht nur so aus, weil sie nämlich so schnell zwischen einzelnen Aufgaben hin und herschalten, dass wir das gar nicht merken. Aber auch der Computerprozessor bewältigt immer nur eine Aufgabe zur gleichen Zeit. Unser Problem ist: Wir haben nur eine sehr begrenzte Kapazität für bewusste Aufmerksamkeit, die nur für einen einzigen bewussten Prozess ausreicht. Versuchen wir zwei Prozesse gleichzeitig durchzuführen, gleichzeitig eine E-Mail lesen und einem Mitarbeiter zuhören etwa, passiert folgen- des: Wir müssen permanent hin und herschalten und uns in der Zwischenzeit im Kurzzeitgedächtnis merken, womit wir gerade zu tun hatten und wozu wir in Kürze zurückzukehren gedenken. Das kostet Ressourcen, Zeit und Energie. Daher ist das gleichzeitige, bewusste Bearbeiten zweier Aufgaben ineffizient. Die kognitive Psychologie hat das intensiv untersucht und kennt das Problem unter dem Namen «switching cost» [1, 2]. Zwei Aufgaben parallel lösen zu wollen, kostet uns mehr Zeit, als sie nacheinander zu bearbeiten. Daher ist Multitasking eine der großen Illusionen des Computerzeitalters und auch einer der großen Zeitfresser. Denn am Ende werden wir dadurch nur langsamer, ineffizienter und grantiger. Wer seine Zeit vermehren will und am Ende dabei glücklicher sein will, dem hilft: jede Sache zu ihrer Zeit, und nur diese. Nichts sonst. Macht zufriedener. Und beschert uns mehr Zeit.

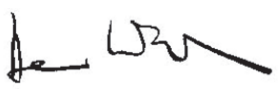

Prof. Dr. Dr. phil. Harald Walach

\section{Literatur}

1 Rubinstein, J. S., Meyer, D. E., \& Evans, J. E.: Executive control of cognitive processes in task switching. Journal of Experimental Psychology: Human Perception and Performance 2001; 27, 763-797.

2 American Psychological Association. (2006). Multitasking: Switching Costs. Retrieved 20.7., 2018, from www.apa.org/research/action/multitask.aspx information@karger.com

www.karger.com/kai

() 2020 S. Karger GmbH, Freiburg

Karger ${ }^{\prime \prime}=$
Prof. Dr. Dr. phil. Harald Walach

CHS Institut

Schönwalder Straße 17, 13347 Berlin, Deutschland

hw@chs-institute.org 